$\mathrm{UK} / 05-08$

hep-th/0508068

\title{
pp Wave Big Bangs: Matrix Strings and Shrinking Fuzzy Spheres
}

\author{
Sumit R. Das* and Jeremy Michelson ${ }^{\dagger}$ \\ Department of Physics and Astronomy \\ University of Kentucky \\ Lexington, $K Y \quad 40506$ \\ U.S.A.
}

\begin{abstract}
We find $p p$ wave solutions in string theory with null-like linear dilatons. These provide toy models of big bang cosmologies. We formulate Matrix String Theory in these backgrounds. Near the big bang "singularity", the string theory becomes strongly coupled but the Yang-Mills description of the matrix string is weakly coupled. The presence of a second length scale allows us to focus on a specific class of non-abelian configurations, viz. fuzzy cylinders, for a suitable regime of parameters. We show that, for a class of $p p$ waves, fuzzy cylinders which start out big at early times dynamically shrink into usual strings at sufficiently late times.
\end{abstract}

*Electronic address: das@pa,uky,edu

${ }^{\dagger}$ Electronic address: jeremy@pa,uky,edu 


\section{Contents}

I. Introduction 2

II. Null Linear Dilaton $p p$ Waves

A. Maximally supersvmmetric $p p$ wave 5

1. Supersvmmetrv 7

B. Linear dilaton dressing of a 24 supercharge $p p$ wave $\quad 7$

1. Supersvmmetrv 8

C. Linear dilaton dressing of the 26 supercharge $p p$ wave 8

D. The nature of the singularity 1

III. Matrix String Theory 9

A. Supersvmmetrv 11

$\begin{array}{ll}\text { IV. Fuzzy Spheres } & 12\end{array}$

V. Giant Gravitons 13

$\begin{array}{ll}\text { VI. Dynamics of Fuzzy Spheres } & 17\end{array}$

$\begin{array}{ll}\text { VII. Fluctuations } & 18\end{array}$

A. $M=$ constant: Fluctuations around the decaving sphere $\quad 19$

B. $M(\tau) \sim e^{Q \tau}$ : Fluctuations around the static spheres $\quad 20$

C. General fluctuations for an irreducible sphere 20

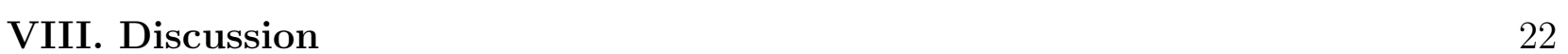

Acknowledgments 23

A. Supersymmetry Transformation of the Matrix String Action 23

$\begin{array}{ll}\text { B. Isometries } & 25\end{array}$

$\begin{array}{ll}\text { References } & 26\end{array}$

\section{INTRODUCTION}

In a recent paper, Craps et. al. [1] proposed a string theoretic toy model of big bang cosmology. The background is the usual flat background of the Type IIA critical superstring with string coupling $g_{s}$ and string length $l_{s}$, living on a compact null direction $x^{-}$with radius $R$

$$
d s^{2}=2 d x^{+} d x^{-}+d \vec{x} \cdot d \vec{x},
$$


and a dilaton linearly proportional to the other null direction $x^{+}$

$$
\Phi=-Q x^{+},
$$

so that the effective string coupling is

$$
\bar{g}_{s}=g_{s} e^{-Q x^{+}} .
$$

The effective string coupling is small for $x^{+} \rightarrow \infty$ for $Q>0$ and one has the usual perturbative spectrum, while for $x^{+} \rightarrow-\infty$ the string theory becomes strongly coupled and the corresponding Einstein metric has a (null-like) big bang singularity. For $Q<0$ we have a time-reversed situation where the big bang is replaced by the big crunch. In this paper we will exclusively deal with $Q>0$. The main observation of [1] is that in the big bang region, the Matrix String description [2, 3, 4] of the theory as a $1+1$ dimensional $U(N)$ Yang Mills theory becomes weakly coupled.

Let us briefly review the Matrix String Theory setup. In the absence of a linear dilaton, the IIA string theory is dual to a IIB string theory with a string coupling $\tilde{g}_{s}$ and string length $\tilde{l}_{s}$ given by

$$
\tilde{g}_{s}=\frac{R}{g_{s} l_{s}}, \quad \tilde{l}_{s}^{2}=\frac{g_{s} l_{s}^{3}}{R} ;
$$

living on a compact space-like circle of radius $\tilde{R}$ given by

$$
\tilde{R}=\frac{l_{s}^{2}}{R} ;
$$

and carrying $N$ units of $D 1$-brane charge. A non-perturbative formulation of the theory is therefore given by a Yang-Mills theory with a dimensional coupling constant

$$
g_{Y M}=\frac{R}{g_{s} l_{s}^{2}} .
$$

For small $g_{s}$, only the Yang-Mills fields belonging to the Cartan subalgebra survive, and the theory describes the original Type IIA string in a light-cone gauge where $x^{+}$is identified with the time $\tau$ of the YM theory.

In the presence of a linear dilaton, it is argued in [1] that the Matrix String Theory may be obtained by simply replacing $g_{s} \rightarrow \bar{g}_{s}$ so that the resulting Yang-Mills theory has a time-dependent coupling which becomes weak as $x^{+} \rightarrow-\infty$. One can equivalently write the theory with a time-independent coupling but on a $1+1$ dimensional Milne universe $[5$, 6, 7, 8, 9, 10, 11, 12, 13, 14, 15, 16, 17, 18, 19, 20] with a compact spatial direction $\sigma$,

$$
d s^{2}=e^{2 Q \tau}\left[-d \tau^{2}+d \sigma^{2}\right] \equiv g_{\alpha \beta} d x^{\alpha} d x^{\beta} .
$$

This models a big bang singularity at $\tau=-\infty$. Since the Yang-Mills theory is weakly coupled at the big bang, all the non-abelian degrees of freedom are important. The key point about this model is that while the "time" of the Yang-Mills theory goes over the 
full range, the space-time as perceived by the bulk string theory with a time-independent coupling has a null-like singularity.

The situation is quite similar to certain time-dependent backgrounds recently studied in two dimensional non-critical string theory 21, 22]. In these latter cases, the fundamental formulation is in terms of Matrix Quantum Mechanics while the closed string theory is described, for example, by collective field theory with space arising out of the eigenvalues. For the backgrounds of [21, 22] the space-time perceived by closed strings are geodesically incomplete with a space-like boundary even though the time of Matrix Quantum Mechanics goes over the full range - provided we use a conformal frame in which the coupling constant of the collective field theory is time-independent. In terms of the string theory there is a space-like condensation of the tachyon. A similar phenomenon occurs in several situations in perturbative string theory, as argued in [23].

Two dimensional non-critical string theory is in a sense solvable. The fundamental degrees of freedom are free fermions and one can use this fact to understand details of what happens near space-like boundaries. Matrix String Theory for critical strings is not as well understood. While it is clear that near the big bang singularity non-abelian degrees of freedom become important, it would be useful to have an idea of how these excitations disappear at late times. This motivated us to look for backgrounds where more is known about non-abelian excitations of matrix theory.

Typically one expects that a certain class of non-abelian excitations of Matrix Theory will play an important role in string or M-theory backgrounds containing fluxes. One example is the fuzzy spheres formed by the dielectric effect [24, 25]. However for most backgrounds, the precise form of the Matrix Theory is not known. Perhaps the most interesting class of backgrounds for which the Matrix Theory is known is provided by M-theory $p p$ waves 26, 27, 28]. For constant dilatons, this matrix theory has been studied extensively [29, 30, 31, 32, 33, 34]. Furthermore, using the compactification procedure of 35], Matrix String Theory has been constructed and studied [36, 37, 38, 39, 40] (see [41] for a different approach). The Matrix String Theory is characterized by a dimensionless combination $M g_{s}$ of the strength of the flux $\mu$, the string coupling $g_{s}$ and the radius $R$,

$$
M g_{s}=\frac{\mu l_{s}^{2}}{R} g_{s} .
$$

When $M g_{s} \ll 1$ the excitations are standard Type IIA strings. For $M g_{s} \gg 1$ the dominant excitations include fuzzy spheres, properties of which have been studied in detail in [33, 40]. (Fuzzy spheres and other membranes in $p p$ wave Matrix Theory and Matrix String Theory have also been studied in $29,30,34,42,43,44,45,46,47]$.)

Note that $M g_{s} \gg 1$ is a weak coupling regime in the Yang-Mills theory [29]. This permits a substantial amount of control over the theory, even though the corrresponding string theory is not perturbative in this domain. In particular, we can use this to explore the region near the initial time singularity. 
In this paper we show that $p p$ wave backgrounds can be easily modified to yield $p p$ waves with null-like linear dilatons, leading to a time dependent coupling constant. Following steps similar to [1] we then write down Matrix String Theories in these backgrounds. As time flows, the parameter which controls the Matrix String Theory flows from weak to strong coupling. Consequently at early times fuzzy spheres dominate the spectrum, while at late times the spectrum consists of ordinary perturbative strings.

Earlier work on cosmology using Matrix Theory include, e.g. 48, 49]. Following the work of [1], other backgrounds with linear dilatons have been studied in [50, 51]. Time-dependent $p p$ wave backgrounds have been studied for a long time. Relevant papers include [52, 53]. In particular, Ref. [52] discussed big bang-type singularities in the context of perturbative strings in $p p$ wave backgrounds with a nontrivial dilaton.

\section{NULL LINEAR DILATON PP WAVES}

In this section we give examples of $p p$ wave solutions of $\mathrm{M}$ theory which lead to string theory backgrounds with null linear dilatons.

\section{A. Maximally supersymmetric $p p$ wave}

Perhaps the simplest and most useful example of $p p$ wave solutions with a linear dilaton arise from the maximally supersymmetric M-theory $p p$ wave. The standard solution of [26] can be easily modified to yield the eleven dimensional background,

$$
\begin{aligned}
d s^{2}= & e^{2 Q x^{+} / 3}\left\{2 d x^{+} d x^{-}-H\left(x^{+}, x^{a}, x^{a^{\prime \prime}}\right)\left(d x^{+}\right)^{2}-\frac{2 \mu\left(x^{+}\right)}{3} e^{-Q x^{+}} x^{8} d x^{9} d x^{+}+\sum_{i=1}^{8}\left(d x^{i}\right)^{2}\right\} \\
& +e^{-4 Q x^{+} / 3}\left(d x^{9}\right)^{2} \\
{ }^{(4)} F= & \mu\left(x^{+}\right) e^{Q x^{+}} d x^{+} \wedge d x^{1} \wedge d x^{2} \wedge d x^{3},
\end{aligned}
$$

where

$$
H\left(x^{+}, x^{a}, x^{a^{\prime \prime}}\right) \equiv\left(\frac{\mu\left(x^{+}\right)}{3}\right)^{2} \sum_{a=1}^{3}\left(x^{a}\right)^{2}+\left(\frac{\mu\left(x^{+}\right)}{6}\right)^{2} \sum_{a^{\prime \prime}=4}^{7}\left(x^{a^{\prime \prime}}\right)^{2},
$$

and we have used indices $a=1,2,3$ and $a^{\prime \prime}=4,5,6,7$. Compactification along $x^{9}$ then yields a IIA solution with a dilaton of the form (1.2).

For $Q=0$, and constant $\mu\left(x^{+}\right)$, this is the maximally supersymmetric M-theory $p p$ wave, written in coordinates amenable to compactification along $x^{9}$ 36, 37, 40]. This remains a solution for $Q \neq 0$ and for any function $\mu\left(x^{+}\right)$. To see this, first note that the four-form squares to zero and is closed. It is also co-closed since the Hodge dual of $d x^{+}$is aligned with $d x^{+}$. 
To verify Einstein's equations it is convenient to choose the elfbein (the caret denotes a tangent space index)

$$
\begin{gathered}
e^{\hat{-}}=e^{Q x^{+} / 3}\left\{d x^{-}-\frac{1}{2}\left[\left(\frac{\mu\left(x^{+}\right)}{3}\right)^{2}\left(x^{a}\right)^{2}+\left(\frac{\mu\left(x^{+}\right)}{6}\right)^{2}\left(x^{a^{\prime \prime}}\right)^{2}+\left(\frac{\mu\left(x^{+}\right)}{3}\right)^{2}\left(x^{8}\right)^{2}\right] d x^{+}\right\}, \\
e^{\hat{+}}=e^{\frac{Q}{3} x^{+}} d x^{+}, \quad e^{\hat{a}}=e^{\frac{Q}{3} x^{+}} d x^{a}, \quad e^{\hat{a}^{\prime \prime}}=e^{\frac{Q}{3} x^{+}} d x^{a^{\prime \prime}}, \quad e^{\hat{8}}=e^{\frac{Q}{3} x^{+}} d x^{8}, \\
e^{\hat{9}}=e^{-2 Q x^{+} / 3} d x^{9}-\frac{\mu\left(x^{+}\right)}{3} e^{Q x^{+} / 3} x^{8} d x^{+} .
\end{gathered}
$$

From this one finds that the nonvanishing components of the Riemann tensor are,

$$
\begin{gathered}
R_{+a+a}=\frac{\mu^{2}\left(x^{+}\right)+Q^{2}}{9} e^{2 Q x^{+} / 3}, \quad R_{+a^{\prime \prime}+a^{\prime \prime}}=\frac{\frac{1}{4} \mu^{2}\left(x^{+}\right)+Q^{2}}{9} e^{2 Q x^{+} / 3}, \quad R_{+8+8}=\frac{\frac{1}{4} \mu^{2}\left(x^{+}\right)+Q^{2}}{9} e^{2 Q x^{+} / 3}, \\
R_{+9+9}=\frac{\frac{1}{4} \mu^{2}\left(x^{+}\right)-8 Q^{2}}{9} e^{-4 Q x^{+} / 3}, \quad R_{+8+9}=\frac{2 \mu\left(x^{+}\right) Q-\mu^{\prime}\left(x^{+}\right)}{6} e^{-Q x^{+} / 3},
\end{gathered}
$$

from which one quickly obtains the sole nonvanishing component of the Ricci tensor

$$
R_{++}=\frac{\mu^{2}\left(x^{+}\right)}{2}=\frac{1}{12} F_{+\mu \nu \rho} F_{+}{ }^{\mu \nu \rho}
$$

so that indeed Einstein's equations are satisfied.

Another way to see that (2.1) is a solution is to consider the Type IIA solution that corresponds to (2.1). The string frame metric, dilaton and Ramond-Ramond (RR) fields are given by

$$
\begin{gathered}
\left.d s^{2}=2 d x^{+} d x^{-}-\left[H\left(x^{+}, x^{a}, x^{a^{\prime \prime}}\right)+\left(\frac{\mu\left(x^{+}\right)}{3}\right)^{2}\left(x^{8}\right)^{2}\right)\right]\left(d x^{+}\right)^{2}+\sum_{i=1}^{8}\left(d x^{i}\right)^{2}, \\
{ }^{(4)} F_{+123}=\mu\left(x^{+}\right) e^{Q x^{+}}, \quad{ }^{(2)} F_{+8}=\frac{\mu\left(x^{+}\right)}{3} e^{Q x^{+}}, \quad \Phi=-Q x^{+} .
\end{gathered}
$$

It is well known that the IIA equations of motion are satisfied for $Q=0$ and for any function $\mu\left(x^{+}\right)$. The equations of motion involve the dilaton in two ways. First there are explicit terms involving derivatives, ${ }^{1} \nabla_{\mu} \nabla_{\nu} \Phi,(\nabla \Phi)^{2}, \nabla^{2} \Phi$. Since the metric has $g^{++}=0$, and the Christoffel symbols have $\Gamma_{\mu \nu}^{+}=0$, it is trivial to see that all these derivative terms vanish. The second class of terms where the dilaton appears are as overall coefficients $e^{2 \Phi}$ multiplying the RR field strength terms in the Einstein equations, namely $e^{2 \Phi}\left({ }^{(4)} F\right)^{2}$ and $e^{2 \Phi}\left({ }^{(2)} F\right)^{2}$. However, the factors of $e^{Q x^{+}}$in the field strengths in (2.6) precisely cancel these coefficients. Therefore the Einstein and dilaton equations reduce precisely to those at $Q=0$. Finally the field strengths are co-closed since they depend only on $x^{+}$and all components $F^{+\mu \nu \alpha}$ or $F^{+\mu}$ vanish. We have compiled the Killing vectors of the IIA background in Appendix B.

\footnotetext{
${ }^{1}$ We use $\mu, \nu, \ldots$ for 10 -dimensional indices; $A, B, \ldots$ for 11 -dimensions and $\alpha, \beta, \ldots$ for $1+1$-dimensions.
} 


\section{Supersymmetry}

It is by now well known [27, 54, 55, 56. that all $p p$ waves are invariant under the sixteen so-called [54] standard supersymmetries that satisfy $\Gamma^{\hat{+}} \epsilon=0$. These supersymmetries are also preserved by the null linear dilaton. Explicitly, the Killing spinor equation is

$$
\mathcal{D}_{A} \epsilon=\nabla_{A} \epsilon-\Omega_{A} \epsilon=0,
$$

where, of course, $\nabla_{A}$ is the usual covariant derivative, and

$$
\Omega_{A}=\frac{1}{24 \cdot 4 !}{ }^{(4)} F_{B C D E}\left(3 \Gamma^{B C D E} \Gamma_{A}-\Gamma_{A} \Gamma^{B C D E}\right) .
$$

For the solution (2.1), the sixteen standard supersymmetries are

$$
\epsilon\left(x^{+}\right)=\exp \left[\frac{Q}{6} x^{+}-\left(\int^{x^{+}} d x \mu(x)\right)\left(\frac{1}{4} \Gamma^{123}+\frac{1}{12} \Gamma^{89}\right)\right] \epsilon_{0}, \quad \Gamma^{+} \epsilon_{0}=0 .
$$

However, it must be emphasized that the standard supersymmetries (2.9) are not interesting. Their existence does not guarantee the supergravity equations of motion, and they are only nonlinearly realized in the Matrix Theory, and on the Green-Schwarz worldsheet.

The interesting supersymmetries are the supernumerary supersymmetries - that is, those supersymmetries that are not standard. For example, the existence of a single supernumerary supersymmetry does guarantee the supergravity equations of motion (a short proof of this can be found in [33]). Also, the supernumerary supersymmetries are linearly realized in the Matrix Theory and on the worldsheet, and are therefore useful for analyzing the spectrum and for nonrenormalization theorems. It is also interesting that toroidal compactification usually breaks some, if not all, of the supernumerary supersymmetry. [35] For example, compactifying the maximally supersymmetric $p p$ wave to the IIA theory breaks eight of the sixteen supernumerary supersymmetries. 36, 37, 40]

Unfortunately, it is also well known that the existence of supernumerary supersymmetries for a $p p$ wave requires that the coefficients in the (Brinkmann) metric be constant. Therefore, there are no supernumerary supersymmetries for the solution (2.1). (Indeed, this is straightforward to verify by considering, say, the integrability condition $\left[\mathcal{D}_{+}, \mathcal{D}_{a}\right]=0$.) Nevertheless, it will turn out that, for a particular choice of $\mu\left(x^{+}\right)$, the Matrix String Theory will have linearly realized supersymmetry currents, which we expect will be useful.

\section{B. Linear dilaton dressing of a 24 supercharge $p p$ wave}

Similar solutions can be written down which have a smaller number of supersymmetries in the $Q=0$ limit. For example, one can consider the Penrose limit of the NS-NS fluxsupported IIA $\mathrm{AdS}_{3} \times \mathrm{S}^{3}$ background. The sixteen supersymmetries of the AdS background are enhanced to 24 upon taking the Penrose limit. This geometry was previously considered 
in [27, 33, 54, 57, 58]. Upon adding the linear dilaton, one obtains the M-theory metric and the four form,

$$
\begin{aligned}
& d s^{2}=e^{2 Q x^{+} / 3}\left[2 d x^{+} d x^{-}-\mu^{2} \sum_{a=1}^{4}\left(x^{a}\right)^{2}\left(d x^{+}\right)^{2}+\sum_{I=1}^{8}\left(d x^{I}\right)^{2}\right]+e^{-4 Q x^{+} / 3}\left(d x^{9}\right)^{2}, \\
& { }^{(4)} F=2 \mu d x^{+} \wedge\left[d x^{1} \wedge d x^{2}+d x^{3} \wedge d x^{4}\right] \wedge d x^{9} .
\end{aligned}
$$

It may be checked that the equations of motion are satisfied. Note that for this $p p$ wave, the linear dilaton does not appear in the field strength; this is related to the fact that the corresponding IIA configuration is supported by NS-NS flux, and not RR flux(es).

\section{Supersymmetry}

Identical comments apply here as in $₫$ II 1 . There are sixteen standard supersymmetries, $\epsilon\left(x^{+}\right)=\exp \left[\frac{Q}{6} x^{+}-\int^{x^{+}} d x \frac{\mu(x)}{2}\left(\Gamma^{129}+\Gamma^{349}\right)\right] \epsilon_{0}, \Gamma^{+} \epsilon_{0}=0$, and no supernumerary supersymmetries for the configuration (2.10), unless $Q=0$ and $\mu$ is constant.

\section{Linear dilaton dressing of the 26 supercharge $p p$ wave}

The nonperturbative physics [33] of the 26 supercharge $p p$ wave [59] is similar to that of the maximally supersymmetric $p p$ wave. However, the corresponding IIA configuration, and therefore the Matrix String Theory, of the 26 supercharge $p p$ wave has two more supercharges than does the (compactification of the) maximally supersymmetric $p p$ wave. One can dress the 26 supercharge $p p$ wave with a dilaton in a way that precisely mimics (2.1). The resulting configuration is,

$$
\begin{gathered}
d s^{2}=e^{2 Q x^{+} / 3}\left\{2 d x^{+} d x^{-}-\mu^{2}\left(x^{+}\right) \sum_{i=1}^{7}\left(x^{i}\right)^{2}\left(d x^{+}\right)^{2}+\sum_{i=1}^{7}\left(d x^{i}\right)^{2}+\left(d x^{8}\right)^{2}\right. \\
\left.+2 \mu\left(x^{+}\right) x^{8} e^{-Q x^{+}} d x^{+} d x^{9}\right\}+e^{-4 Q x^{+} / 3}\left(d x^{9}\right)^{2} \\
{ }^{(4)} F=\mu\left(x^{+}\right) e^{Q x^{+}} d x^{+} \wedge\left[-3 d x^{1} \wedge d x^{2} \wedge d x^{3}-\sum_{y=1}^{3} d x^{y} \wedge \omega_{y}^{-}\right]
\end{gathered}
$$

where $\omega_{y}^{-}$are the anti-selfdual two-forms of the $\mathbb{R}^{4}$ spanned by $x^{4 \cdots 7}$.

\section{The nature of the singularity}

For the configurations introduced in this section, the dilaton blows up at $x^{+}=-\infty$. Since $g^{++}=0$, the surface $x^{+}=-\infty$ is null-like. The geodesic equations include

$$
\frac{d^{2} x^{+}(\lambda)}{d \lambda^{2}}=-\frac{2 Q}{3}\left(\frac{d x^{+}(\lambda)}{d \lambda}\right)^{2}
$$


where $\lambda$ is the affine parameter, so that either $x^{+}=$constant or

$$
x^{+}(\lambda)=\frac{3}{2 Q} \ln \lambda,
$$

(by choice of affine parameterization). This demonstrates that the null singularity at $x^{+}=-\infty$ is reached in finite affine parameter, (and that reaching $x^{+}=\infty$ requires infinite affine parameter, and is therefore not a singularity.) These conclusions are precisely as in [1] - and, in fact, are independent of $\mu$ !

Let us also point out that all time-like geodesics start at the singularity. For example, for the $p p$ wave (2.1), the only geodesics that do not hit the singularity have the very simple form

$$
x^{+}=x_{0}^{+}, \quad x^{i}=x_{0}^{i}+p^{i} \lambda, \quad i \neq 9, \quad x^{9}=x_{0}^{9}, \quad x^{-}=x_{0}^{-}+p^{-} \lambda+\frac{Q}{6}\left(p^{i}\right)^{2} \lambda^{2},
$$

which is never timelike, and null iff $p^{i}=0$-and still independent of $\mu$.

\section{MATRIX STRING THEORY}

Following 1] we propose that the action of Matrix String Theory in the null linear dilaton $p p$ wave backgrounds may be obtained from the usual action by replacing the string coupling $g_{s}$ by $\bar{g}_{s}$ defined in (1.3). The resulting action, for the $p p$ wave (2.1), is $(i, j=1 \ldots 8)$

$$
\begin{aligned}
S=\int d \tau d \sigma \operatorname{Tr}\{ & \frac{1}{2} \bar{g}_{s}^{2} F_{\tau \sigma}^{2}+\frac{1}{2}\left(D_{\tau} X^{i}\right)^{2}-\frac{1}{2}\left(D_{\sigma} X^{i}\right)^{2}+\frac{1}{4 \bar{g}_{s}^{2}}\left[X^{i}, X^{j}\right]^{2} \\
& -\frac{1}{2}\left(\frac{M(\tau)}{3}\right)^{2}\left(X^{a}\right)^{2}-\frac{1}{2}\left(\frac{M(\tau)}{6}\right)^{2}\left(X^{a^{\prime \prime}}\right)^{2} \\
& \left.-\frac{M(\tau)}{3} \bar{g}_{s} X^{8} F_{\tau \sigma}-i \frac{M(\tau)}{3 \bar{g}_{s}} \epsilon_{a b c} X^{a} X^{b} X^{c}\right\}+S_{\text {fermion }} .
\end{aligned}
$$

where $M(\tau)$ is given in terms of $\mu(\tau)$ by

$$
M(\tau)=\frac{\mu(\tau) l_{s}^{2}}{R} .
$$

The worldsheet time $\tau$ has to be identified with $x^{+}$.

The fermionic part of the action is

$$
S_{\text {fermion }}=\int d \tau d \sigma \operatorname{Tr}\left\{-i \bar{\Psi} \not \Psi \Psi+\frac{1}{\bar{g}_{s}} \bar{\Psi} \rho^{3} \Gamma^{i}\left[X^{i}, \Psi\right]-i \frac{M}{4} \bar{\Psi} \rho^{3}\left(\Gamma^{123}-\frac{1}{3} \Gamma^{89}\right) \Psi\right\},
$$

where $\Psi$ is a $1+1$ dimensional Majorana fermion, each Weyl component of which is an $U(N)$ matrix-valued Majorana-Weyl $\mathrm{SO}(8)$ spinor. The $\mathrm{SO}(8)$ chirality is opposite to the $\mathrm{SO}(1,1)$ chirality. ${ }^{2}$ The $\Gamma$ 's are $\mathrm{SO}(8)$ Dirac matrices, the $\rho$ 's are $\mathrm{SO}(1,1)$ Dirac matrices, and $D \equiv \rho^{\tau} D_{\tau}+\rho^{\sigma} D_{\sigma}$.

\footnotetext{
${ }^{2}$ We use $\rho^{3}=-\rho^{0} \rho^{1}$.
} 
We emphasize that we have not derived the above matrix model using some kind of Seiberg-Sen 60, 61] argument. (Indeed, the accepted derivation [29] of the $Q=0$ Matrix Model is still suspect because of the use of a non-spacelike circle, whereas the whole point of the Seiberg-Sen argument is to rephrase the null-like compactification in terms of a spacelike one. But it is not obvious how to fix that derivation.)

The bosonic terms in the above action may be, however, obtained by considering the lightcone gauge action of a single massless particle in 11 dimensions with momentum $p_{-}=N / R$ (or equivalently the action for $N$ D0 branes in the IIA theory) following [24, 25, 62]. The lagrangian of the matrix theory may be written as

$$
\begin{aligned}
L=\operatorname{Tr} & \left\{\frac{1}{2}\left(R G^{+-}\right) G_{I J} D_{\tau} X^{I} D_{\tau} X^{J}-\frac{1}{R} G_{I J} G^{-I} D_{\tau} X^{J}+\frac{1}{2 G^{+-} R}\left(G_{I J} G^{-I} G^{-J}-G^{--}\right)\right. \\
& \left.+\frac{R}{4 G^{+-}} G_{I K} G_{J L}\left[X^{I}, X^{J}\right]\left[X^{K}, X^{L}\right]-\frac{i}{2} A_{+I J} X^{I} X^{J}\right\}
\end{aligned}
$$

where $G$ denotes components of the eleven dimensional metric and $I, J=1 \cdots 9$ denote all the transverse directions. In writing (3.4) we have used the fact that $G^{++}=0$ and that the only nonvanishing components of the 3 -form potential are of the form $A_{+I J}$. Using the metric and the potential from (2.1) this becomes

$$
\begin{aligned}
L=R \operatorname{Tr} & \left\{\frac{1}{2 R^{2}}\left(D_{\tau} X^{i}\right)^{2}+\frac{1}{2 R^{2}} e^{-2 Q \tau}\left(D_{\tau} X^{i}\right)^{2}-\frac{\mu}{3 R^{2}} e^{-Q \tau} X^{8} D_{\tau} X^{9}\right. \\
& -\frac{1}{2}\left(\frac{\mu}{3 R}\right)^{2}\left(X^{a}\right)^{2}-\frac{1}{2}\left(\frac{\mu}{6 R}\right)^{2}\left(X^{a}\right)^{2} \\
& \left.+\frac{1}{4} e^{2 Q \tau}\left[X^{i}, X^{j}\right]^{2}+\frac{1}{2}\left[X^{9}, X^{i}\right]^{2}-i \frac{\mu}{3 R} \epsilon_{a b c} e^{Q \tau} X^{a} X^{b} X^{c}\right\} .
\end{aligned}
$$

Simultaneous compactification and T-dualization along $X^{9}$ means we introduce the spatial direction $\sigma$ and identify

$$
X^{9}=i \hat{R} D_{\sigma}, \quad \operatorname{Tr} \rightarrow \int_{0}^{2 \pi} d \sigma, \quad D_{\tau} X^{9}=-\hat{R} F_{\tau \sigma} .
$$

A standard set of rescalings then leads to (3.1). These steps are identical to those in [40] and will not be repeated here. In a later section we will see that a comparison of the dynamics of giant gravitons and those of fuzzy spheres provides an important check on our guess.

To understand the nature of the big bang singularity in this model it is useful to rewrite the action as if it had the background metric (1.7). This effectively removes the $\tau$-dependence from the string coupling. In this form the action is

$$
\begin{aligned}
& S=S_{\text {fermion }}+\int d \tau d \sigma \sqrt{-g} \operatorname{Tr}\left\{-\frac{1}{4} g_{s}^{2} F_{\alpha \beta}^{2}-\frac{1}{2}\left(D_{\alpha} X^{i}\right)^{2}+\frac{1}{4 g_{s}^{2}}\left[X^{i}, X^{j}\right]^{2}\right. \\
& \left.-\frac{1}{2}\left(\frac{\tilde{M}(\tau)}{3}\right)^{2}\left(X^{a}\right)^{2}-\frac{1}{2}\left(\frac{\tilde{M}(\tau)}{6}\right)^{2}\left(X^{a^{\prime \prime}}\right)^{2}-\frac{\tilde{M}(\tau)}{6} g_{s} X^{8} \epsilon^{\alpha \beta} F_{\alpha \beta}-i \frac{\tilde{M}(\tau)}{3 g_{s}} \epsilon_{a b c} X^{a} X^{b} X^{c}\right\}
\end{aligned}
$$


where we have defined

$$
\tilde{M}(\tau) \equiv M(\tau) e^{-Q \tau}
$$

Upon rescaling the fermions $\Psi_{\text {new }}=e^{-Q \tau / 2} \Psi_{\text {old }}$ (as for a conformal transformation) one has

$$
S_{\text {fermion }}=\int d \tau d \sigma \sqrt{-g} \operatorname{Tr}\left\{i \bar{\Psi} \not D \Psi+\frac{1}{g_{s}} \bar{\Psi} \rho^{3} \Gamma^{i}\left[X^{i}, \Psi\right]-i \frac{\tilde{M}(\tau)}{4} \bar{\Psi} \rho^{3}\left(\Gamma^{123}-\frac{1}{3} \Gamma^{89}\right) \Psi\right\} .
$$

In this form, we have a $1+1$ dimensional Yang-Mills theory with a time-dependent mass for the scalars and constant coupling, defined on a space-time with metric $g_{\alpha \beta}$ given in (1.7).

\section{A. Supersymmetry}

The Matrix String action has nonlinearly realized supersymmetry corresponding to the standard supersymmetries. This is not interesting.

Generically, the action has no linearly realized supersymmetries (see Appendix A]). This is not surprising, since the supergravity background has no supernumerary supersymmetries. The supersymmetry variation of the action has two terms. The first, which we call the bulk term, is an integral over $\sigma, \tau$, while the second is a boundary term. At finite times, both these terms are non-vanishing - the boundary term is non-vanishing, as in [1], because of non-trivial boundary conditions on fermions in the $\sigma$ direction. The integrand of the bulk term however, vanishes exponentially fast at late times while at the same time the radius of the $\sigma$ circle also increases exponentially fast. This means that in some sense supersymmetry is restored at late times.

Interestingly, for the special case,

$$
\mu(\tau)=\mu_{0} e^{Q \tau}
$$

the Matrix String Theory has 8 linearly realized supercurrents corresponding to the transformations

$$
\begin{gathered}
\delta X^{i}=i \bar{\Psi} \rho^{3} \Gamma^{i} \epsilon, \\
\delta \Psi=\frac{1}{4} F_{\alpha \beta} \rho^{\alpha \beta} \epsilon-\frac{1}{2} \epsilon^{\alpha}{ }_{\beta} D_{\alpha} X^{i} \Gamma^{i} \rho^{\beta} \epsilon+\frac{i}{4}\left[X_{\alpha} \epsilon, X^{j}\right] \Gamma^{i j} \epsilon \\
+\frac{\tilde{M}(\tau)}{6} X^{a} \Gamma^{a} \Gamma^{123} \epsilon-\frac{\tilde{M}(\tau)}{12} X^{a^{\prime \prime}} \Gamma^{a^{\prime \prime}} \Gamma^{123} \epsilon,
\end{gathered}
$$

where

$$
\epsilon=\Gamma^{4567} \epsilon_{0}=\Gamma^{12389} \epsilon_{0}
$$

Note that for $Q \neq 0$, these supercurrents cannot be integrated, because of the nonvanishing boundary term. (The boundary terms are given in Eq. (A.6e).) Of course, for $Q=0$ 
these do yield supersymmetries that are precisely those given and discussed in [40]. For the special case (3.10) however, the string frame metric of the Type IIA theory evolves from flat space at early times to a space-time whose geodesics at late times are effectively those of a two-dimensional space-time, since the ++ component of the metric provides a harmonic restoring force towards $x^{i}=0$ which becomes infinite as $x^{+} \rightarrow \infty .^{3}$

\section{FUZZY SPHERES}

Let us recall the ground states of the $p p$ wave Matrix string theory for $Q=0$ and constant $\mu(\tau)=\mu$. As shown in [40], one can rescale the coordinates and the fields by

$$
\tau \rightarrow M \tau, \quad \sigma \rightarrow M \sigma, \quad A_{\mu} \rightarrow A_{\mu} / M, \quad X^{i} \rightarrow X^{i} /\left(M g_{s}\right), \quad \Psi \rightarrow \Psi /\left(M^{3 / 2} g_{s}\right)
$$

to bring out an overall factor of $\left(M g_{s}\right)^{2}$ out of the action, with no other factor of $M$ or $g_{s}$ inside. This shows that the quantity $M g_{s}$ is the inverse coupling constant of the theory. For $M g_{s} \ll 1$ the theory is strongly coupled, and only fields which lie in the Cartan subalgebra survive. By a gauge choice we can eliminate the gauge field and choose the $X^{i}$ to be diagonal; the remaining gauge freedom allows boundary conditions as we go around the $\sigma$ direction that correspond to multi-string states. This leads to usual perturbative IIA theory.

For $M g_{s} \gg 1$, the dominant configurations are obtained by solving the classical equations of motion and the lowest energy states include static fuzzy spheres. In terms of the original variables, these solutions are given by

$$
X^{a}=\frac{M g_{s}}{3} J^{a}, \quad(a=1,2,3),
$$

where $J^{a}$ are generators of a $N$-dimensional representation of $S U(2)$ algebra. Note that these are independent of $\sigma$; they should be called "fuzzy cylinders" in the YM theory. As discussed in [40], small fluctuations around this ground state have an equally spaced spectrum of $N^{2}$ degrees of freedom whose boundary conditions along the $\sigma$ direction correspond to fuzzy cylinders that do not generically close upon going around the $\sigma$ circle.

The rescaling in (4.1) can be performed in the matrix model in (3.1) as well (note the quantity that appears in the rescaling is $g_{s}$ and not $\bar{g}_{s}$ ). However now various terms in the action have explicit time dependence. Thus $M g_{s} \gg 1$ does not guarantee that classical solutions dominate for all times. In fact it is clear from (3.1) that as $\tau \rightarrow \infty$ the coefficients of the terms which involve commutators of $X$ grow large, so that the fields $X^{i}$ are more and more constrained to lie in the Cartan subalgebra even when $M g_{s} \gg 1$. It may be easily

\footnotetext{
3 This discussion, however, is for the sector of the theory with constant $P_{-}=N / R$. The coordinate change $y^{+}=e^{Q x^{+}} / Q, y^{-}=e^{-Q x^{+}} x^{-}$, results in the metric $d s^{2}=2 d y^{+} d y^{-}-\left[\left(\frac{\mu_{0}}{3}\right)^{2}\left(x^{a}\right)^{2}+\left(\frac{\mu_{0}}{6}\right)^{2}\left(x^{a^{\prime \prime}}\right)^{2}+\left(\frac{\mu_{0}}{3}\right)^{2}\left(x^{8}\right)^{2}+2 \frac{y^{-}}{y^{+}}\right]\left(d y^{+}\right)^{2}+\left(d x^{i}\right)^{2}, \quad$ which $\quad$ asymptotes at large $y^{+}$to the usual $p p$ wave.
} 
checked that in this case the matrix string action reduces to the standard Green-Schwarz light cone worldsheet action of a string in this background. Our aim to is investigate the dynamics of these fuzzy spheres as the geometry evolves from the big bang.

\section{GIANT GRAVITONS}

In the $N=\infty$ limit of the $Q=0$ background, the fuzzy cylinders of the Matrix String Theory become giant gravitons [63, 64, 65, 66, 67, 68]. In the IIA description these are $D 2$ branes with quantized momenta $p_{-}=N / R$ along $x^{-}$, while in the M-theory lift they are $M 2$ branes with this momentum. To get an idea of the nature of excitations of string theory for $Q \neq 0$ it is useful to re-examine the dynamics of these $M 2$ branes in the background given by (2.1). We will do the analysis for a general $\mu\left(X^{+}\right)$.

We will use polar coordinates $(r, \theta, \phi)$ in the plane defined by the Cartesian coordinates $x^{a}, a=1 \cdots 3$. In these coordinates the field strength is

$$
F_{+r \theta \phi}=\mu\left(X^{+}\right) e^{Q X^{+}} r^{2} \sin \theta,
$$

and we can choose a gauge such that the potential is

$$
C_{+\theta \phi}=\frac{1}{3} \mu\left(X^{+}\right) e^{Q X^{+}} r^{3} \sin \theta,
$$

with all other components zero.

The M2 brane is wrapped around the $(\theta, \phi)$ direction. This allows us to fix a gauge in which the spatial coordinates on the worldvolume are identified with the angles $\theta$ and $\phi$. We want to restrict our dynamics to the sector where the remaining coordinates are independent of $\theta, \phi$. This is a consistent truncation which respects the equations of motion. Then all worldvolume fields depend only on the worldvolume time $\tau$ and the action of the M2 brane reduces to that of a point particle:

$$
S=4 \pi T_{2} \int d \tau\left[-e^{2 Q X^{+} / 3} r^{2}(\tau) \sqrt{-G_{A B} \partial_{\tau} X^{A} \partial_{\tau} X^{B}}+\frac{\mu\left(X^{+}\right)}{3} e^{Q X^{+}} r^{3}(\tau) \partial_{\tau} X^{+}\right],
$$

where $A, B$ stand for $\left(r, X^{a^{\prime \prime}}, X^{8}, X^{9}, X^{ \pm}\right)$. The metric $G_{A B}$ is the solution given in (2.1).

The canonical momenta are given by

$$
P_{A}=4 \pi T_{2}\left[\frac{e^{2 Q X^{+} / 3}}{\sqrt{-G_{A B} \partial_{\tau} X^{A} \partial_{\tau} X^{B}}} r^{2} G_{A B} \partial_{\tau} X^{B}+\frac{\mu\left(X^{+}\right) r^{3}}{3} e^{Q X^{+}} \delta_{A}^{+}\right] .
$$

The identity

$$
G^{A B}\left[P_{A}-\frac{4 \pi T_{2}}{3} \mu\left(X^{+}\right) e^{Q X^{+}} r^{3} \delta_{A}^{+}\right]\left[P_{B}-\frac{4 \pi T_{2}}{3} \mu\left(X^{+}\right) e^{Q X^{+}} r^{3} \delta_{B}^{+}\right]=-\left(4 \pi T_{2} r^{2}\right)^{2} e^{4 Q X^{+} / 3},
$$

yields the dispersion relation. 
The above gauge choice still allows arbitrary reparametrization of the worldvolume time coordinate $\tau$. We fix this by choosing a gauge $\tau=X^{+}$so that the canonical Hamiltonian is given by $H=-P_{+}$.

Since $X^{-}$and $X^{9}$ are isometry directions, $P_{-}$and $P_{9}$ are conserved quantities. We will consider the sector of the theory in which $P_{9}=0$. The momenta $P_{a^{\prime \prime}}$ and $P_{8}$ are not conserved. However it is easy to see that $P_{8}=X^{8}=P_{a^{\prime \prime}}=0$ is a solution of the equations of motion. Furthermore, in this sector $X^{a^{\prime \prime}}=X^{9}=0$ is a consistent solution. The Hamiltonian of the truncated problem then simplifies considerably and may be written as

$$
H=\frac{P_{r}^{2}}{2 P_{-}}+\frac{\left(4 \pi T_{2}\right)^{2}}{2 P_{-}} e^{2 Q \tau}\left(\frac{\mu(\tau) P_{-}}{12 \pi T_{2}} r e^{-Q \tau}-r^{2}\right)^{2},
$$

where we have used the explicit form of the metric (2.1). This is the Hamiltonian of a particle with mass $P_{-}$moving in a time-dependent potential. The equation of motion is

$$
P_{-}^{2} \frac{d^{2} r}{d \tau^{2}}+\left(4 \pi T_{2}\right)^{2} e^{2 Q \tau}\left(\frac{\mu(\tau) P_{-}}{12 \pi T_{2}} r e^{-Q \tau}-r^{2}\right)\left(\frac{\mu(\tau) P_{-}}{12 \pi T_{2}} e^{-Q \tau}-2 r\right)^{2} .
$$

For $Q=0$ and constant $\mu$, the lowest energy solution is static with

$$
r=r_{0}=\frac{\mu}{12 \pi T_{2}} P_{-} .
$$

This is the giant graviton solution discussed in [40].

For $Q \neq 0$ it is useful to introduce the variables

$$
\mathcal{R}(t) \equiv \frac{12 \pi T_{2}}{\mu(\tau) P_{-}} r(\tau) e^{Q \tau}, \quad t=Q \tau
$$

The equation (5.7) then becomes

$\frac{d^{2} \mathcal{R}}{d t^{2}}-2 \frac{d \mathcal{R}}{d t}\left(1-\frac{1}{2} \frac{A^{\prime}(t)}{A(t)}\right)+\mathcal{R}\left(1-\frac{A^{\prime}(t)}{A(t)}-\frac{A^{\prime}(t)^{2}}{4 A(t)^{2}}+\frac{A^{\prime \prime}(t)}{2 A(t)}\right)+A(t)(1-2 \mathcal{R})\left(\mathcal{R}-\mathcal{R}^{2}\right)=0$,

where

$$
A(t) \equiv\left(\frac{\mu(\tau)}{3 Q}\right)^{2},
$$

is the single parameter which enters into this equation.

Let us first examine the nature of the solutions to (5.10) for constant $\mu$, i.e. a constant $A$. In this case the differential equation (5.10) reads

$$
\frac{d^{2} \mathcal{R}}{d t^{2}}-2 \frac{d \mathcal{R}}{d t}+\mathcal{R}\left[(1+A)-3 A \mathcal{R}+2 A \mathcal{R}^{2}\right]=0
$$

As this equation has no explicit $t$-dependence, it is easy to see that for some range of the values of $A$ there is a solution with constant $\mathcal{R}=\mathcal{R}_{0}$

$$
\mathcal{R}_{0}=0, \frac{3}{4} \pm \sqrt{\frac{1}{16}-\frac{1}{2 A}}
$$




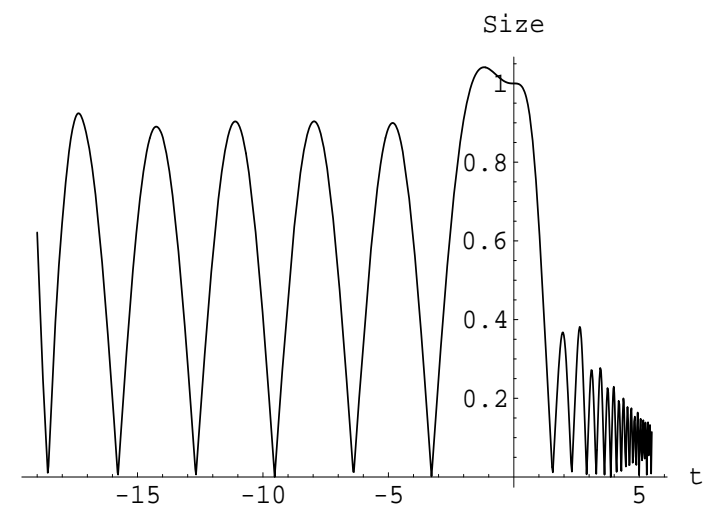

FIG. 1: The scaled size of a giant graviton as a function of the time $t$ for $A=1$.

which are all real for $A>8$. This means that the radius of the giant graviton $r(\tau)$ has the behavior

$$
r(\tau)=\frac{\mu P_{-} \mathcal{R}_{0}}{12 \pi T_{2}} e^{-Q \tau}
$$

In other words, at early times the giant gravitons are big, while at late times they shrink to zero size at an exponential rate. We see that for large $A$, this roughly reproduces (upon choosing the "+" root) (5.8), in the sense that one obtains

$$
r(t)=\frac{\mu P_{-}}{12 \pi T_{2}}\left[\frac{3}{4}+\frac{1}{4} \sqrt{1-8 A^{-1}}\right] e^{-Q \tau}=\frac{\mu P_{-}}{12 \pi T_{2}} e^{-Q \tau}+\mathcal{O}\left(A^{-1}\right) .
$$

For $A<8$ the roots become complex and there are no nonzero constant $\mathcal{R}$ solutions.

Note that (5.14) is an exact solution and not an adiabatic approximation. However this corresponds to special initial conditions. Generically, it is not hard to see that if one starts out with a M2 brane of finite size at early times, it will collapse to zero size at late times exponentially, regardless of the value of $A .{ }^{4}$ Fig. 1 shows a numerical solution of this equation for $A=1$, plotted in terms of the scaled size of the giant graviton

$$
\rho(\tau)=\mathcal{R}(\tau) e^{-Q \tau},
$$

with initial conditions $\rho(0)=1$ and $\dot{\rho}(0)=0$, (i.e. a typical size at a time when the coupling is $\left.\mathcal{O}\left(g_{s}\right)\right) . \quad \rho(t)$ oscillates in $t$, but the amplitude goes down at large $t$. This means that at late times the size goes to zero essentially because of the fact that in equation (5.7) the potential becomes infinitely steep. This behavior is typical for other initial conditions - the behavior at early times depends on the initial conditions but the amplitude always decreases to zero at large times. For smaller values of $A$ the size of the M2 brane remains big for a longer time period, but the late time behavior is universal. This is illustrated in Fig. [2]

\footnotetext{
${ }^{4}$ As a crude approximation, one can use a "perturbative expansion" and ignore the higher powers of $\mathcal{R}$ in Eq. (5.12). Then the solutions are $\mathcal{R}(t) \sim \exp [(-1 \pm i \sqrt{A}) t]$, demonstrating that the fuzzy spheres are falling off even faster than for large $A$, and oscillate with a period of order $A^{-1 / 2}$.
} 


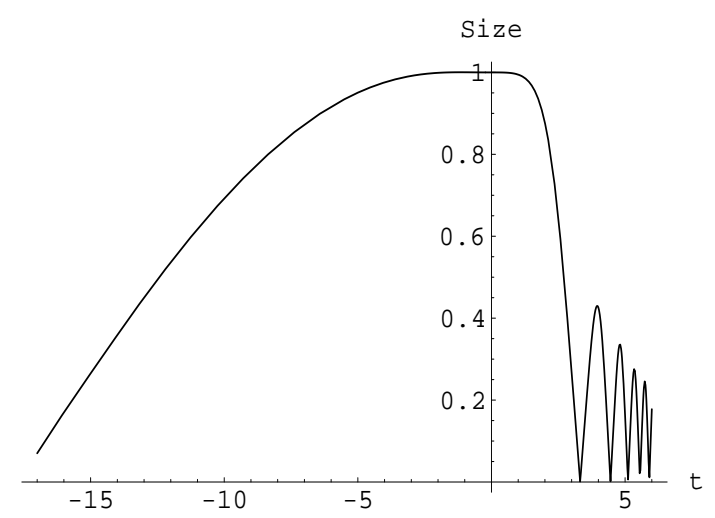

FIG. 2: Same as Fig. 1, but with $A=0.01$. Extended to smaller times, the size appears to hit a zero and then rise rapidly as time is further decreased; however, this is a regime for which our numerical solution is unreliable.

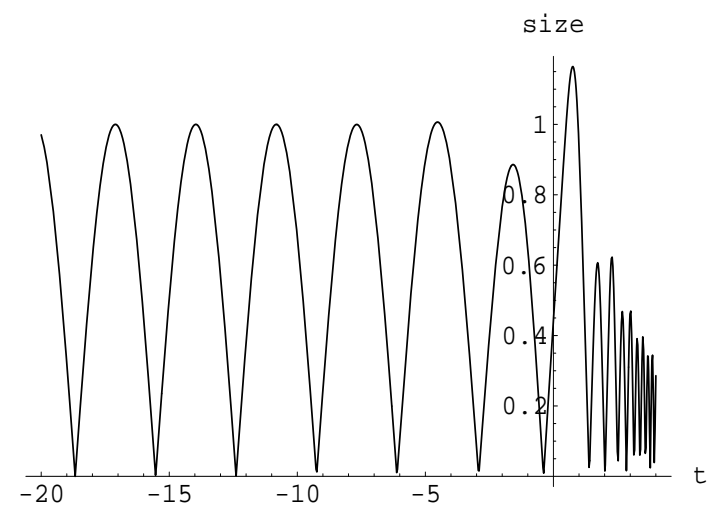

FIG. 3: Similar to Fig. 1] with $A=1$, but initial conditions at an earlier time.

Alternatively one could impose final state conditions demanding that the size of the giant graviton vanishes at sufficiently late time. As expected, typically the amplitudes of oscillations of the size increases as we go back in time. One can also demand that the giant graviton have a typical size at an earlier time. This is depicted in Figs. 3] and 4. One continues to see that the size of the giant graviton falls off at late times.

Let us now discuss some features of the solution for a time-dependent $\mu(\tau)$. We have not investigated this in full generality. However it is easy to see that when $\mu(\tau)=\mu e^{Q \tau}$, then one quickly finds static solutions

$$
r(\tau)=r_{0}=0, \frac{\mu P_{-}}{12 \pi T_{2}}, \frac{\mu P_{-}}{24 \pi T_{2}} .
$$

In fact the first and second solutions have vanishing energies at all times, just as for $Q=0$ with constant $\mu$. For generic initial conditions, a numerical solution shows that the size is attracted to either $r=0$ or towards $r=r_{0}$ at late times. 


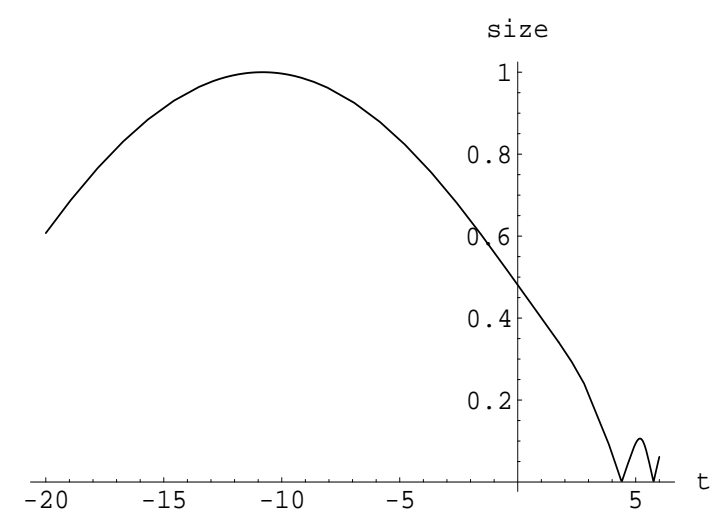

FIG. 4: Same as Fig. 3, but with $A=0.01$.

\section{DYNAMICS OF FUZZY SPHERES}

The dynamics of fuzzy sphere configurations is similar to that of giant gravitons. To examine the simplest situation consider the matrix string action in the sector

$$
X^{4}=X^{5}=\cdots=X^{8}=0, \quad A_{\mu}=0, \quad \Psi=0 .
$$

This restriction is consistent with the equations of motion. The action then takes a simple form

$$
S=\int d \tau d \sigma \operatorname{Tr}\left\{\frac{1}{2}\left(\partial_{\tau} X^{a}\right)^{2}-\frac{1}{2}\left(\partial_{\sigma} X^{a}\right)^{2}+\frac{1}{4 \bar{g}_{s}^{2}}\left(\left[X^{a}, X^{b}\right]-i \frac{M(\tau) \bar{g}_{s}}{3} \epsilon^{a b}{ }_{c} X^{c}\right)^{2}\right\} .
$$

We will look at $\sigma$-independent configurations of the form

$$
X^{a}(\sigma, \tau)=S(\tau) J^{a}, \quad(a=1,2,3),
$$

where $J^{a}$ is an $N$-dimensional representation of $S U(2)$. When $Q=0$ this configuration would be a classical solution with a constant $S=M g_{s} / 3$ - this is the fuzzy cylinder of the IIB theory carrying a D1 brane charge, which becomes a fuzzy sphere of the original IIA theory carrying a momentum $p_{-}$along $x^{-}$. When $J^{a}$ is the irreducible representation we have a single fuzzy sphere whereas a $J^{a}$ in a reducible representation corresponds to multiple fuzzy spheres.

When $Q \neq 0$ a constant $S$ would not be a solution, and we would like to find how $S$ evolves in time. For $J^{a}$ in the $N$-dimensional irreducible representation,

$$
\operatorname{Tr} \sum_{a}\left(J^{a}\right)^{2}=\frac{N\left(N^{2}-1\right)}{4}
$$

and the action (6.2) becomes

$$
S=\frac{N\left(N^{2}-1\right)}{8} \int d \tau d \sigma\left[\left(\frac{d S}{d \tau}\right)^{2}-\frac{1}{2 \bar{g}_{s}^{2}}\left(S^{2}-\frac{M(\tau) \bar{g}_{s}}{3} S\right)^{2}\right] .
$$


Using the variables

$$
\xi(t)=\frac{3}{M(\tau) g_{s}} S(\tau) e^{Q \tau}, \quad t \equiv Q \tau,
$$

it is straightforward to see that the equation of motion becomes

$$
\frac{d^{2} \xi}{d t^{2}}-2 \frac{d \xi}{d t}\left(1-\frac{1}{2} \frac{B^{\prime}(t)}{B(t)}\right)+\xi\left(1-\frac{B^{\prime}(t)}{B(t)}-\frac{B^{\prime}(t)^{2}}{4 B(t)^{2}}+\frac{B^{\prime \prime}(t)}{2 B(t)}\right)+B(t)(1-2 \xi)\left(\xi-\xi^{2}\right)=0,
$$

where

$$
B(t) \equiv\left(\frac{M(t)}{3 Q}\right)^{2}
$$

This is identical in form to (5.10). Therefore the time dependence of the radius of the fuzzy spheres $S(\tau)$ is identical to that of the radius of giant gravitons in the previous section.

The fact that the dynamics of these fuzzy spheres is in exact agreement with the dynamics of giant gravitons is an important check that our guess for the Matrix string action - in particular the way the various factors of $e^{Q \tau}$ appear in (3.1) - is indeed correct.

\section{FLUCTUATIONS}

In the previous sections we found two simple exact solutions of the equations of motion for giant gravitons, or equivalently fuzzy spheres, in the irreducible representation of $S U(2)$ at large $\mathrm{N}$. The first solution appears when $\mu(\tau)$ is a constant, and has a sphere which contracts exponentially in time. In the Matrix String Theory definitions of fields, the classical size of the fuzzy sphere is given by

$$
S(\tau)=\frac{M g_{s}}{3} \xi_{0} e^{-Q \tau},
$$

where

$$
\xi_{0}=\frac{3}{4}+\sqrt{\frac{1}{16}-\frac{1}{B}}
$$

and $B$ is given by (6.8). The second solution appears when $\mu(\tau)$ or equivalently $M(\tau)$ has the special time dependence

$$
M(\tau)=M_{0} e^{Q \tau} .
$$

This is the case where the supersymmetry of the action is broken only by boundary conditions. In this case there is a specific solution where the size of the sphere is constant in time, given by

$$
S_{0}=\frac{M_{0} g_{s}}{3}
$$

To examine the physics of these time-dependent solution we need to determine the dynamics of small fluctuations around these solutions. We have not performed a complete analysis of these fluctuations. However the dynamics of a special class of fluctuations can be found from what we have already done. These are fluctuations of the form

$$
X^{a}(\sigma, \tau)=\left[S_{0}(\tau)+\varphi(\sigma, \tau)\right] J^{a},
$$


with all other fields set to zero. In the language of giant gravitons this means that we are looking at fluctuations which are spherically symmetric on the worldvolume. In this section we will determine the spectrum around the two special solutions (7.1) and (7.4).

\section{A. $\quad M=$ constant: Fluctuations around the decaying sphere}

The equations of motion of quadratic fluctuations around (17.1) can be written down by considering perturbations of the equations (6.7) supplemented by a $\sigma$-derivative term. These become simple in terms of the variables

$$
\tilde{\varphi}=\frac{3}{M g_{s}} e^{Q \tau} \varphi,
$$

and the equation for $\tilde{\varphi}$ is

$$
\frac{d^{2} \tilde{\varphi}}{d t^{2}}-\frac{1}{Q^{2}} \frac{d^{2} \tilde{\varphi}}{d \sigma^{2}}-2 \frac{d \tilde{\varphi}}{d t}+\nu^{2} \tilde{\varphi}=0,
$$

where we have used $t=Q \tau$, and

$$
\nu^{2}=A \xi_{0}\left(4 \xi_{0}-3\right)
$$

Note that (77.2) implies that $\nu^{2}>0$. The modes of the form

$$
\tilde{\varphi} \sim e^{-i \omega t+i k Q \sigma},
$$

have dispersion relation

$$
\omega=i \pm \sqrt{k^{2}+\nu^{2}-4} .
$$

The imaginary part comes from the anti-damping term in the equation (6.7). The quantity in the square root may be calculated to be

$$
\beta^{2}=k^{2}+\nu^{2}-4=\frac{1}{4}\left[4 k^{2}+12 B \sqrt{\frac{1}{16}-\frac{1}{B}}+16 B-24\right] .
$$

This is explicitly positive since our solution holds only for $B>8$. The imaginary part of $\omega$, however means that the solutions for $\tilde{\varphi}$ grow with time. This growth is exactly compensated by the fact that $\tilde{\varphi}$ defined in (7.6) already contains a factor of $e^{t}$, so that the fluctuations of the radius itself, $\varphi$ behaves as

$$
\varphi \sim e^{i k \sigma} e^{ \pm i \frac{\beta}{2}}
$$

which are standard positive and negative frequency oscillator modes.

This seems to suggest that even though the background fuzzy spheres shrink to zero size at late times, the non-abelian excitations consisting of spherically symmetric fluctuations of the size of these spheres have standard oscillatory behavior, corresponding to usual particles. However, the non-linear terms involving $\varphi$ become large at late times. In fact it is easy to see that the terms which are of order $\varphi^{3}$ come with an overall coefficient of $e^{Q \tau}$ while those which are $O\left(\varphi^{4}\right)$ have an overall coefficient of $e^{2 Q \tau}$. Therefore at late times these terms will constrain $\varphi$ to vanish. Therefore we conclude at late times, not only do the fuzzy spheres shrink to zero size, but the amplitudes of breathing modes vanish as well. 


\section{B. $M(\tau) \sim e^{Q \tau}$ : Fluctuations around the static spheres}

The situation is quite different for the case of time dependent $M$ given by (17.3). In this case it is easier to analyze the situation from the original action (6.2) restricted to (77.5). The quadratic action for $\varphi$ may be easily seen to be

$$
S=\int d \tau d \sigma\left[\frac{1}{2}\left(\partial_{\tau} \varphi\right)^{2}-\frac{1}{2}\left(\partial_{\sigma} \varphi\right)^{2}-\frac{1}{2}\left(\frac{M_{0}}{3}\right)^{2} e^{2 Q \tau} \varphi^{2}\right] .
$$

This is the action of a field with time-dependent mass in two dimensions. By a standard conformal transformation, this is equivalent to the action of a standard massive field in the Milne quadrant.

The physics for such a field is quite well known [69, 70]. In Milne time, the in and out vacua are inequivalent. This leads to particle production. If we start out with vacuum at the big bang one ends up with a thermal state at late times. For our problem, the reverse situation is more interesting. If we demand that the state at late times is the vacuum of the $\varphi$ particles, we have to start out with a thermal state at the big bang.

\section{General fluctuations for an irreducible sphere}

The general problem of small fluctuations can be treated as in [40]. We consider this not just to reanalyze the results of the previous subsections in more generality, but also to address the question - especially considering the growth of the fluctutations - of whether the truncation of the problem to (6.2) is physically reasonable. For the standard $p p$ wave $-Q=0$ and constant $\mu$-fuzzy spheres minimize the energy. This is also true for the static spheres. For the general problem however, the spheres are not static, and so the corresponding solution to the equations of motion sets neither the potential energy nor the kinetic energy to zero. We would therefore like to at least check that the solutions we have been discussing are (perturbatively) stable. That is, we check that there are no negative modes in the spectrum of small oscillations about the solution.

Upon expanding the fields in terms of matrix spherical harmonics, ${ }^{5}$

$$
\begin{gathered}
X^{a}=S(\tau) J^{a}+\sum_{j, l, m} x_{j l m} Y_{j l m}^{a} \\
X^{a^{\prime \prime}}=\sum_{l m} x_{l m}^{a^{\prime \prime}} Y_{l m}, \quad X^{8}=\sum_{l m} x_{l m}^{8} Y_{l m}, \quad A_{\alpha}=\sum_{l m} a_{\alpha l m} Y_{l m}
\end{gathered}
$$

\footnotetext{
${ }^{5}$ We will attempt to be brief. Further details on matrix scalar and vector spherical harmonics can be found in [40]. These details include the range of summation, and reality properties of the fluctuating fields in the expansion.
} 
one quickly finds that the dynamics of the fluctuations $x_{l m}^{a^{\prime \prime}}$ are governed by the action

$$
S=\int d \tau d \sigma\left\{\left|\dot{x}_{l m}^{a^{\prime \prime}}\right|^{2}-\left|x_{l m}^{a^{\prime \prime}}\right|^{2}-\left[\frac{l(l+1) S(\tau)^{2}}{\bar{g}_{s}^{2}}+\left(\frac{M(\tau)}{6}\right)^{2}\right]\left|x_{l m}^{a^{\prime \prime}}\right|^{2}\right\} .
$$

Note that these are positive "mass" fluctuations. For the static fuzzy spheres, the mass is precisely that of [40], dressed with the time-dependence of the Milne universe.

Similarly, the fluctuations $x_{l \pm 1, l, m}$ are described by

$$
\begin{aligned}
& S=\int d \tau d \sigma\left\{\left|\dot{x}_{l+1, l, m}\right|^{2}-\left|x_{l+1, l, m}^{\prime}\right|^{2}-\frac{1}{2 \bar{g}_{s}^{2}}\left(\frac{M(\tau) \bar{g}_{s}}{3}+S(\tau) l\right)^{2}\left|x_{l+1, l m}\right|^{2}\right\}, \\
& S=\int d \tau d \sigma\left\{\left|\dot{x}_{l-1, l, m}\right|^{2}-\left|x_{l-1, l, m}^{\prime}\right|^{2}-\frac{1}{2 \bar{g}_{s}^{2}}\left(\frac{M(\tau) \bar{g}_{s}}{3}-S(\tau)(l+1)\right)^{2}\left|x_{l-1, l, m}\right|^{2}\right\} .
\end{aligned}
$$

These are again positive, and, for the static sphere, are the Milne universe analogs of the results of [40]. The radial fluctuation of $S(\tau)$ itself is encoded in $x_{010}$ (cf. (17.13)).

The remaining fluctuations are coupled in a rather complicated way. The analysis is performed, as in [40], by introducing auxiliary scalar fields $\phi_{l m}$ which facilitate integrating out the gauge field. The result is independent of the gauge degrees of freedom $x_{l l m}$. The case of $l=0$ is special, and we temporarily defer that interesting discussion. For $l \neq 0$, one finds the action

$$
\begin{aligned}
S=\int d \tau d \sigma & \left\{\left|\dot{x}_{l m}^{8}\right|^{2}-\left|x_{l m}^{\prime 8}\right|^{2}+\frac{1}{S(\tau)^{2} l(l+1)}\left[\left|\frac{\partial}{\partial \tau}\left(\omega_{l} \bar{g}_{s} \phi_{l m}\right)\right|^{2}-\omega_{l}^{2} \bar{g}_{s}^{2}\left|\phi_{l m}^{\prime}\right|^{2}\right]\right. \\
& \left.-\left[\frac{M(\tau)^{2}}{9}+\frac{S(\tau)^{2}}{\bar{g}_{s}^{2}} l(l+1)\right]\left|X_{l m}^{8}\right|^{2}+\frac{M(\tau)}{3} \omega_{l}\left(\phi_{l m}^{*} X_{l m}^{8}+\phi_{l m} X_{l m}^{8 *}\right)\right\} .
\end{aligned}
$$

Here $\omega_{l}$ is the so-far unspecified and arbitrary (possibly time-dependent) normalization of $\phi_{l m}$. Note that, unlike the $p p$ wave considered in [40], the problem appears to be nontrivial to untangle. However, it is clear that the mass matrix is positive-definite, as is the kinetic term, so this suggests that the solution is stable. That said, the friction term in the equation of motion for $\phi$ could result in a growth of fluctuations. Finally, let us make our now-familiar comment that, for the static spheres, upon choosing the normalization $\omega_{l}=\frac{M(\tau)}{3} \sqrt{l(l+1)}$ the masses again precisely reproduce the Milne universe analogues of the masses of [40].

For $l=0$, the result is quite simple,

$$
S=\int d \tau d \sigma\left\{\left|\dot{x}_{00}^{8}\right|^{2}-\left|x_{00}^{\prime 8}\right|^{2}-\left|\frac{M(\tau)}{3} X_{00}^{8}-\omega_{l} \phi_{00}\right|^{2}\right\}, \quad \omega_{l} \bar{g}_{s} \phi_{00}=\text { const. }
$$

Thus, for static fuzzy spheres, it is natural to choose $\omega_{l}=\frac{M(\tau)}{3}$. This implies $\phi_{00}=$ const, and allows us to preserve a minimum energy by taking $X_{00}^{8}=\phi_{00}=$ const. These are precisely the solutions of [40] which corresponded, upon lifting back up to 11-dimensions, to "orbiting" fuzzy spheres. Their 11-dimensional interpretation for $Q \neq 0$ is less clear. 
Finally, let us make a comment about constant $\mu$ and $M$. In this case, $S(\tau)$ falls off exponentially like $e^{-Q \tau}$ at late times. Therefore, the fluctuations $x^{a^{\prime \prime}}$ and $x_{l \pm 1, l, m}$ asymptote to fluctuations in terms of a constant mass, as was found in \$VIIA Upon choosing $\omega_{l}=$ $\bar{g}_{s}^{-1} \sqrt{l(l+1)}$, one similarly finds a constant mass matrix for $x_{l m}^{8}$ and $\phi_{l m}(l \neq 0)$ but a nontrivial (damping!) kinetic term for the $\phi_{l m}$. The zero mode of $X^{8}$, however, loses its shift symmetry.

\section{DISCUSSION}

As argued in [1], in the class of backgrounds considered in this paper, one would expect non-abelian configurations of Matrix String Theory to be important at early times, while at late times, the excitations are dominated by usual strings. We have shown that $p p$ wave geometries provide a useful laboratory to investigate the detailed time evolution of a class of non-abelian configurations. The presence of another length scale in the problem, viz. the strength of the background flux $\mu$, leads to a natural class of such non-abelian configuration spheres. The picture is most transparent for constant $\mu$. When $M g_{s} \gg 1$ classical configurations dominate the dynamics. The classical dynamics is controlled by a single combination of the background flux $\mu$ and the rate of change of the dilaton $Q$, viz. $B=M /(3 Q)$. For large enough $B$, there is a special class of solutions for which the time dependence of the radius is a pure exponential. At early times, when the IIA theory is strongly coupled, the radii are big while they go to zero exponentially at late times. Generic time dependent solutions have oscillatory behavior; the radii generically oscillate, but with amplitudes which go to zero at late times. This means that at late times only diagonal matrices survive, which lead to usual perturbative strings. While we expect that this is generically true for all non-abelian configurations, we do not have any definitive results for this.

One interesting feature of fuzzy sphere configurations of usual $p p$ wave matrix string theory is the fact that the fluctuations around the classical solutions correspond to fields which do not generically return to their original values as we go around the compact circle any number of times. It would be interesting to see how these reduce to usual periodic strings as the size of the fuzzy spheres go to zero at late times.

It is clear from our discussion that $p p$ wave solutions with linear null dilatons may be easily obtained in Type IIB theory, e.g. for the Penrose limit of $A d S_{5} \times S^{5}$. The behavior of giant gravitons in such backgrounds may be investigated along the lines of this paper. However in this case the bulk geometry has a different holographic description in terms of the large R-charge limit of $N=4$ Yang-Mills theory and giants can be identified as specific states in this gauge theory. It would be interesting to investigate the implications of a linear null dilaton for the Yang-Mills theory. 


\section{Acknowledgments}

We thank M. Berkooz, B. Craps, C. Gomez, P. Mukhopadhyay, A. Shapere, S. Trivedi and C. Zhou for useful conversations. This work was supported in part by Department of Energy contract \#DE-FG01-00ER45832 and the National Science Foundation grant No. PHY-0244811. S.R.D. would like to thank Tata Institute of Fundamental Research for hospitality and the organizers of the Benasque String Theory Workshop for providing a wonderful atmosphere.

\section{APPENDIX A: SUPERSYMMETRY TRANSFORMATION OF THE MATRIX STRING ACTION}

In this appendix we give a general formula for the supersymmetry transformation of the action (3.1).

Since, for $\mu=0$, supersymmetry is manifest in flat coordinates, we perform the coordinate transformation [1]

$$
\pm \tau+\sigma= \pm \frac{1}{Q} \ln \left(\sqrt{2} Q \xi^{ \pm}\right), \quad \xi^{ \pm}=\frac{1}{\sqrt{2}}\left(\xi^{0} \pm \xi^{1}\right) .
$$

Then the Matrix String action is

$$
\begin{aligned}
& S=S_{\text {fermion }}+\int d^{2} \xi \operatorname{Tr}\left\{-\frac{1}{4} g_{s}^{2} F_{\alpha \beta}^{2}-\frac{1}{2}\left(D_{\alpha} X^{i}\right)^{2}+\frac{1}{4 g_{s}^{2}}\left[X^{i}, X^{j}\right]^{2}\right. \\
& \left.-\frac{1}{2}\left(\frac{\tilde{M}(\xi)}{3}\right)^{2}\left(X^{a}\right)^{2}-\frac{1}{2}\left(\frac{\tilde{M}(\xi)}{6}\right)^{2}\left(X^{a^{\prime \prime}}\right)^{2}-\frac{\tilde{M}(\xi)}{6} g_{s} X^{8} \epsilon^{\alpha \beta} F_{\alpha \beta}-i \frac{\tilde{M}(\xi)}{3 g_{s}} \epsilon_{a b c} X^{a} X^{b} X^{c}\right\}
\end{aligned}
$$

where

$$
\tilde{M}(\xi)=\frac{\mu(\xi) l_{s}^{2}}{Q R \xi}, \quad \xi \equiv \sqrt{2 \xi^{+} \xi^{-}}=\frac{1}{Q} e^{Q \tau}
$$

For simplicity, we have written $\mu(\xi)$ instead of $\mu(\tau(\xi))$. In contrast to the main text, we use nonchiral, Majorana $\mathrm{SO}(8)$ fermions $\Psi$, and lose manifest $\mathrm{SO}(1,1)$ covariance, so that

$$
\begin{aligned}
S_{\text {fermion }}=\int d^{2} \xi \operatorname{Tr}\left\{i \Psi^{\top} D_{0} \Psi-i \Psi^{\top} \Gamma^{9} D_{1} \Psi\right. & \\
& \left.+\frac{1}{g_{s}} \Psi^{\top} \Gamma^{i}\left[X^{i}, \Psi\right]-i \frac{\tilde{M}(\xi)}{4} \Psi^{\top}\left(\Gamma^{123}-\frac{1}{3} \Gamma^{89}\right) \Psi\right\} .
\end{aligned}
$$

When $\mu$, and therefore $M$, is constant, and $Q=0$, the action has eight linearly realized supersymmetries parametrized by $\epsilon_{0}=\Gamma^{4567} \epsilon_{0}=\Gamma^{12389} \epsilon_{0} \cdot{ }^{6}$ In overcomplete generality, one

\footnotetext{
${ }^{6}$ The last expression is the statement that we use the convention $\Gamma^{123456789}=\mathbb{1}$.
} 
can consider the supersymmetry transformations

$$
\begin{gathered}
\delta X^{i}=i \alpha_{1}(\xi) \Psi^{\top} \Gamma^{i} \epsilon, \quad \delta A_{0}=i \alpha_{3}(\xi) \Psi^{\top} \epsilon, \quad \delta A_{1}=i \alpha_{2}(\xi) \Psi^{\top} \Gamma^{9} \epsilon \\
\delta \Psi=\frac{1}{2} \alpha_{4}(\xi) F_{01} \Gamma^{9} \epsilon+\frac{1}{2} \alpha_{5}(\xi) D_{0} X^{i} \Gamma^{i} \epsilon-\frac{1}{2} \alpha_{6}(\xi) D_{1} X^{i} \Gamma^{i} \Gamma^{9} \epsilon+\frac{i}{4} \alpha_{7}(\xi)\left[X^{i}, X^{j}\right] \Gamma^{i j} \epsilon \\
+\alpha_{8}(\xi) \frac{\tilde{M}(\xi)}{6} X^{a} \Gamma^{a} \Gamma^{123} \epsilon-\alpha_{9}(\xi) \frac{\tilde{M}(\xi)}{12} X^{a^{\prime \prime}} \Gamma^{a^{\prime \prime}} \Gamma^{123} \epsilon,
\end{gathered}
$$

where

$$
\epsilon=\Gamma^{4567} \epsilon_{0}=\Gamma^{12389} \epsilon_{0}
$$

For $Q=0, \tilde{M}(\xi)$ constant and $\alpha_{\alpha}(\xi)=1$, this reduces to the supersymmetry transformations given in [40], and preserves the action. In the current context, the variation of the action is

$$
\delta S=\delta S_{\mathrm{t} . \mathrm{d} .}+\delta S_{1}+\delta S_{\partial}+\delta S_{\tilde{M}}
$$

where the various pieces of the action are

$$
\begin{aligned}
& \delta S_{1}=\int d \xi^{0} d \xi^{1} \operatorname{Tr}\left\{i\left(\alpha_{1}-\alpha_{4}\right) \frac{\tilde{M}}{3} F_{01} \epsilon^{\top} \Gamma^{8} \Psi+i\left(\alpha_{2}-\alpha_{4}\right) F_{01} \epsilon^{\top} D_{1} \Psi\right. \\
& -i\left(\alpha_{3}-\alpha_{4}\right) F_{01} \epsilon^{\top} \Gamma^{9} D_{0} \Psi+\left(\frac{\alpha_{5}+\alpha_{6}}{2}-\alpha_{4}\right)\left[F_{01}, X^{i}\right] \epsilon^{\top} \Gamma^{i} \Gamma^{9} \Psi+i \frac{\alpha_{5}-\alpha_{6}}{2}\left\{D_{0}, D_{1}\right\} \epsilon^{\top} \Gamma^{i} \Gamma^{9} \Psi \\
& +\frac{i}{3}\left(\alpha_{8}-\alpha_{5}\right) \tilde{M} D_{0} X^{a} \epsilon^{\top} \Gamma^{a} \Gamma^{123} \Psi+\frac{i}{6}\left(\alpha_{9}-\alpha_{5}\right) \tilde{M} D_{0} X^{a^{\prime \prime}} \epsilon^{\top} \Gamma^{a^{\prime \prime}} \Gamma^{123} \Psi \\
& -\frac{i}{3}\left(\alpha_{3}-\alpha_{5}\right) \tilde{M} D_{0} X^{8} \epsilon^{\top} \Gamma^{9} \Psi+i\left(\alpha_{1}-\alpha_{8}\right)\left(\frac{\tilde{M}}{3}\right)^{2} X^{a} \epsilon^{\top} \Gamma^{a} \Psi \\
& +i\left(\alpha_{1}-\alpha_{9}\right)\left(\frac{\tilde{M}}{6}\right)^{2} X^{a^{\prime \prime}} \epsilon^{\top} \Gamma^{a^{\prime \prime}} \Psi+\frac{i}{3}\left(\alpha_{6}-\alpha_{8}\right) \tilde{M} D_{1} X^{a} \epsilon^{\top} \Gamma^{a} \Gamma^{123} \Gamma^{9} \Psi \\
& +\frac{i}{6}\left(\alpha_{6}-\alpha_{9}\right) \tilde{M} D_{1} X^{a^{\prime \prime}} \epsilon^{\top} \Gamma^{a^{\prime \prime}} \Gamma^{123} \Gamma^{9} \Psi-\frac{i}{3}\left(\alpha_{6}-\alpha_{2}\right) \tilde{M} D_{1} X^{8} \epsilon^{\top} \Psi \\
& -i\left(\alpha_{1}-\alpha_{5}\right) D_{0} X^{i} \epsilon^{\top} \Gamma^{i} D_{0} \Psi+i\left(\alpha_{1}-\alpha_{6}\right) D_{1} X^{i} \epsilon^{\top} \Gamma^{i} D_{1} \Psi \\
& +\left(\alpha_{5}-\alpha_{2}\right) D_{0} X^{i} \epsilon^{\top}\left[X^{i}, \Psi\right]+\left(\alpha_{5}-\alpha_{7}\right) D_{0} X^{i} \epsilon^{\top} \Gamma^{i j}\left[X^{j}, \Psi\right] \\
& +\left(\alpha_{3}-\alpha_{6}\right) D_{1} X^{i} \epsilon^{\top} \Gamma^{9}\left[X^{i}, \Psi\right]+\left(\alpha_{7}-\alpha_{6}\right) D_{1} X^{i} \epsilon^{\top} \Gamma^{i j} \Gamma^{9}\left[X^{j}, \Psi\right] \\
& -\frac{\tilde{M}}{3}\left(\alpha_{8}+\frac{1}{2} \alpha_{7}-\frac{3}{2} \alpha_{1}\right)\left[X^{a}, X^{b}\right] \epsilon^{\top} \Gamma^{a b} \Gamma^{123} \Psi+\frac{\tilde{M}}{3}\left(\alpha_{8}-\frac{1}{2} \alpha_{9}-\frac{1}{2} \alpha_{7}\right)\left[X^{a}, X^{b^{\prime \prime}}\right] \epsilon^{\top} \Gamma^{a b^{\prime \prime}} \Gamma^{123} \Psi \\
& +\frac{\tilde{M}}{3}\left(\alpha_{8}-\alpha_{7}\right)\left[X^{a}, X^{8}\right] \epsilon^{\top} \Gamma^{a 8} \Gamma^{123} \Psi+\frac{\tilde{M}}{6}\left(\alpha_{9}-\alpha_{7}\right)\left[X^{a^{\prime \prime}}, X^{b^{\prime \prime}}\right] \epsilon^{\top} \Gamma^{a^{\prime \prime} b^{\prime \prime}} \Gamma^{123} \Psi \\
& \left.+\frac{\tilde{M}}{6}\left(\alpha_{9}-\alpha_{7}\right)\left[X^{a^{\prime \prime}}, X^{8}\right] \epsilon^{\top} \Gamma^{a^{\prime \prime} 8} \Gamma^{123} \Psi+i\left(\alpha_{1}-\alpha_{7}\right)\left[\left[X^{i}, X^{j}\right], X^{j}\right] \epsilon^{\top} \Gamma^{i} \Psi\right\} ; \\
& \delta S_{\partial}=\int d \xi^{0} d \xi^{1} \operatorname{Tr}\left\{i \partial_{1} \alpha_{2} F_{01} \epsilon^{\top} \Psi-i \partial_{0} \alpha_{3} F_{01} \epsilon^{\top} \Gamma^{9} \Psi-i \partial_{0} \alpha_{6} D_{1} X^{i} \epsilon^{\top} \Gamma^{i} \Gamma^{9} \Psi\right. \\
& +i \partial_{1} \alpha_{5} D_{0} X^{i} \epsilon^{\top} \Gamma^{i} \Gamma^{9} \Psi-i \partial_{0} \alpha_{1} D_{0} X^{i} \epsilon^{\top} \Gamma^{i} \Psi+i \partial_{1} \alpha_{1} D_{1} X^{i} \epsilon^{\top} \Gamma^{i} \Psi \\
& \left.-\frac{1}{2} \partial_{0} \alpha_{7}\left[X^{i}, X^{j}\right] \epsilon^{\top} \Gamma^{i j} \Psi+\frac{1}{2} \partial_{1} \alpha_{7}\left[X^{i}, X^{j}\right] \epsilon^{\top} \Gamma^{i j} \Gamma^{9} \Psi\right\} ;
\end{aligned}
$$




$$
\begin{aligned}
& \delta S_{\tilde{M}}=\int d \xi^{0} d \xi^{1} \operatorname{Tr}\left\{\frac{i}{6} \partial_{0}\left(\alpha_{9} \tilde{M}\right) X^{a^{\prime \prime}} \epsilon^{\top} \Gamma^{a^{\prime \prime}} \Gamma^{123} \Psi-\frac{i}{3} \alpha_{3} \partial_{0} \tilde{M} X^{8} \epsilon^{\top} \Gamma^{9} \Psi\right. \\
&+\frac{i}{3} \partial_{0}\left(\alpha_{8} \tilde{M}\right) X^{a} \epsilon^{\top} \Gamma^{a} \Gamma^{123} \Psi-\frac{i}{3} \partial_{1}\left(\alpha_{8} \tilde{M}\right) X^{a} \epsilon^{\top} \Gamma^{a} \Gamma^{123} \Gamma^{9} \Psi \\
&\left.-\frac{i}{6} \partial_{1}\left(\alpha_{9} \tilde{M}\right) X^{a^{\prime \prime}} \epsilon^{\top} \Gamma^{a^{\prime \prime}} \Gamma^{123} \Gamma^{9} \Psi+\frac{i}{3} \alpha_{2} \partial_{1} \tilde{M} X^{8} \epsilon^{\top} \Psi\right\} ;
\end{aligned}
$$

and the total derivatives are

$$
\begin{aligned}
\delta S_{\text {t.d. }} & =\int d^{2} \xi \operatorname{Tr}\left\{\partial _ { 0 } \left[\frac{i}{2} \alpha_{6} D_{1} X^{i} \epsilon^{\top} \Gamma^{i} \Gamma^{9} \Psi-\frac{i}{6} \alpha_{8} \tilde{M} X^{a} \epsilon^{\top} \Gamma^{a} \Gamma^{123} \Psi-\frac{i}{12} \alpha_{9} \tilde{M} X^{a^{\prime \prime}} \epsilon^{\top} \Gamma^{a^{\prime \prime}} \Gamma^{123} \Psi\right.\right. \\
- & \left.\frac{i}{3} \alpha_{3} \tilde{M} X^{8} \epsilon^{\top} \Gamma^{8} \Gamma^{123} \Psi+\frac{1}{4} \alpha_{7}\left[X^{i}, X^{j}\right] \epsilon^{\top} \Gamma^{i j} \Psi-\frac{i}{2} \alpha_{4} F_{01} \epsilon^{\top} \Gamma^{9} \Psi-\frac{i}{2} \alpha_{5} D_{0} X^{i} \epsilon^{\top} \Gamma^{i} \Psi\right] \\
& +\partial_{1}\left[\frac{i}{2} \alpha_{5} D_{0} X^{i} \epsilon^{\top} \Gamma^{i} \Gamma^{9} \Psi+\frac{i}{6} \alpha_{8} \tilde{M} X^{a} \epsilon^{\top} \Gamma^{a} \Gamma^{123} \Gamma^{9} \Psi+\frac{i}{12} \alpha_{9} \tilde{M} X^{a^{\prime \prime}} \epsilon^{\top} \Gamma^{a^{\prime \prime}} \Gamma^{123} \Gamma^{9} \Psi\right. \\
+ & \left.\left.\frac{i}{3} \alpha_{2} \tilde{M} X^{8} \epsilon^{\top} \Gamma^{8} \Gamma^{123} \Gamma^{9} \Psi-\frac{1}{4} \alpha_{7}\left[X^{i}, X^{j}\right] \epsilon^{\top} \Gamma^{i j} \Gamma^{9} \Psi+\frac{i}{2} \alpha_{4} F_{01} \epsilon^{\top} \Psi+\frac{i}{2} \alpha_{6} D_{1} X^{i} \epsilon^{\top} \Gamma^{i} \Psi\right]\right\} .
\end{aligned}
$$

The coefficients of the various combinations of fields in Eq. (A.6b) vanish only if the $\alpha$ 's are equal. Eq. (A.6C) then yields that the $\alpha$ 's should be constant, whereupon Eq. (A.6d) then implies that $\tilde{M}$ should be constant as well. That is, there are supersymmetry currents when the bulk part of the integrand vanishes. This happens (only, by choice of normalization) for $\alpha_{1}=\alpha_{2}=\cdots=\alpha_{9}=1$ and for $\xi$-independent $\tilde{M}$. This clearly occurs for the maximally supersymmetric $p p$ wave, but also occurs by taking $\mu(\xi) \propto \xi \propto e^{Q \tau}$. Of course, as in [1], even in that case, the total derivatives (A.6e) contribute because of the nontrivial topology of the Milne universe.

\section{APPENDIX B: ISOMETRIES}

It is interesting to work out the Killing vectors of the IIA background (2.6). We present those here - see also [52] - and mimic the notation of [55].

The obvious Killing vectors are the null isometry,

$$
e_{-}=-\frac{\partial}{\partial x^{-}}
$$

and the rotational Killing vectors,

$$
J_{a b}=x^{a} \frac{\partial}{\partial x^{b}}-x^{b} \frac{\partial}{\partial x^{a}}, \quad \quad J_{a^{\prime \prime} b^{\prime \prime}}=x^{a^{\prime \prime}} \frac{\partial}{\partial x^{b^{\prime \prime}}}-x^{b^{\prime \prime}} \frac{\partial}{\partial x^{a^{\prime \prime}}} .
$$

The less obvious Killing vectors are (no sum)

$$
\begin{aligned}
e_{i} & =k_{i}^{(1)}\left(x^{+}\right) \frac{\partial}{\partial x^{i}}-x^{i} \partial_{+} k_{i}^{(1)}\left(x^{+}\right) \frac{\partial}{\partial x^{-}} \\
e_{i}^{*} & =k_{i}^{(2)}\left(x^{+}\right) \frac{\partial}{\partial x^{i}}-x^{i} \partial_{+} k_{i}^{(2)}\left(x^{+}\right) \frac{\partial}{\partial x^{-}}
\end{aligned}
$$


where $k_{i}^{(1)}$ and $k_{i}^{(2)}$ are the two solutions to the second order differential equation,

$$
0= \begin{cases}\partial_{+}^{2} k_{i}\left(x^{+}\right)+\left(\frac{\mu\left(x^{+}\right)}{3}\right)^{2} k_{i}\left(x^{+}\right), & i=1,2,3,8 \\ \partial_{+}^{2} k_{i}\left(x^{+}\right)+\left(\frac{\mu\left(x^{+}\right)}{6}\right)^{2} k_{i}\left(x^{+}\right), & i=4,5,6,7\end{cases}
$$

For constant $\mu$, these are precisely the Killing vectors previously given in the literature (e.g. 55]) and for the case $\mu\left(x^{+}\right)=\mu e^{Q x^{+}}$, one has, in terms of Bessel and Neumann functions,

$$
k_{i}^{(1)}\left(x^{+}\right)=J_{0}\left(\hat{\xi}_{i}\right), \quad k_{i}^{(2)}\left(x^{+}\right)=N_{0}\left(\hat{\xi}_{i}\right), \quad \hat{\xi}_{i}= \begin{cases}\frac{\mu}{3 Q} \xi, & i=1,2,3,8 \\ \frac{\mu}{6 Q} \xi, & i=4,5,6,7\end{cases}
$$

The isometry algebra is therefore 8 copies of the Heisenberg algebra, with a common central element, proportional to $e_{-}$with a normalization that is given by the (constant!) Wronskian of the two solutions to the differential equation (B.4 $)$.

[1] B. Craps, S. Sethi and E. P. Verlinde, A matrix big bang, hep-th/0506180.

[2] L. Motl, Proposals on nonperturbative superstring interactions, hep-th/9701025.

[3] T. Banks and N. Seiberg, Strings from matrices, Nucl. Phys. B497 (1997) 41; [hep-th/9702187].

[4] R. Dijkgraaf, E. P. Verlinde and H. L. Verlinde, Matrix string theory, Nucl. Phys. B500 (1997) 43; [hep-th/9703030].

[5] G. T. Horowitz and A. R. Steif, Space-time Singularities in String Theory, Phys. Rev. Lett. 64 (1990) 260.

[6] V. Balasubramanian, S. F. Hassan, E. Keski-Vakkuri and A. Naqvi, A space-time orbifold: A toy model for a cosmological singularity, Phys. Rev. D 67 (2003) 026003; [hep-th/0202187].

[7] L. Cornalba and M. S. Costa, A new cosmological scenario in string theory, Phys. Rev. D 66 (2002) 066001; [hep-th/0203031].

[8] J. Simon, The geometry of null rotation identifications, J. High Energy Phys. 06 (2002) 001; [hep-th/0203201].

[9] H. Liu, G. Moore and N. Seiberg, String in a time-dependent orbifold, J. High Energy Phys. 06 (2002) 045; [hep-th/0204168].

[10] H. Liu, G. Moore and N. Seiberg, String in time-dependent orbifolds, J. High Energy Phys. 10 (2002) 031; [hep-th/0205288].

[11] A. J. Tolley and N. Turok, Quantum Fields in a Big Crunch/Big Bang Spacetime, Phys. Rev. D 66 (2002) 106005; [hep-th/0204091].

[12] B. Craps, D. Kutasov and G. Rajesh, String propagation in the presence of cosmological singularities, J. High Energy Phys. 06 (2002) 053; [hep-th/0205101].

[13] M. Fabinger and J. McGreevy, On smooth time-depedent orbifolds and null singularities, J. High Energy Phys. 06 (2003) 042; [hep-th/0206196].

[14] J. R. David, Plane waves with weak singularities, J. High Energy Phys. 11 (2003) 064; [hep-th/0303013]. 
[15] B. C. Da Cunha and E. J. Martinec, Closed string tachyon condensation and worldsheet inflation, Phys. Rev. D 68 (2003) 063502 [hep-th/0303087].

[16] J. G. Russo, Cosmological string models from Milne spaces and $S L(2, \mathbb{Z})$ orbifold, Mod. Phys. Lett. $\boldsymbol{A 1 9}$ (2004) 421-432; [hep-th/0305032].

[17] A. Giveon, E. Rabinovici and A. Sever, Strings in singular time-dependent backgrounds, Fortsch. Phys. 51 (2003) 805-823; [hep-th/0305137].

[18] B. Pioline and M. Berkooz, Strings in an electric field, and the Milne universe, J. Cosmol. Astropart. Phys. 11 (2003) 007; [hep-th/0307280].

[19] B. Durin and B. Pioline, Closed strings in Misner space: A toy model for a big bounce?, LPTHE-P05-03, hep-th/0501145.

[20] M. Berkooz, B. Durin, B. Pioline and D. Reichmann, Closed strings in Misner space: Stringy fuzziness with a twist, J. Cosmol. Astropart. Phys. 10 (2004) 002; hep-th/0407216.

[21] S. R. Das and J. L. Karczmarek, Spacelike boundaries from the $c=1$ matrix model, Phys. Rev. D 71 (2005) 086006; [hep-th/0412093].

[22] S. R. Das, Non-trivial 2d space-times from matrices, hep-th/0503002.

[23] J. McGreevy and E. Silverstein, The tachyon at the end of the universe, hep-th/0506130.

[24] R. C. Myers, Dielectric-branes, J. High Energy Phys. 12 (1999) 022; [hep-th/9910053].

[25] W. Taylor and M. Van Raamsdonk, Multiple Dp-branes in Weak Background Fields, Nucl. Phys. B573 (2000) 703-734; [hep-th/9910052].

[26] D. Berenstein, J. M. Maldacena and H. Nastase, Strings in flat space and pp waves from $N=$ 4 super Yang Mills, J. High Energy Phys. 04 (2002) 013; [hep-th/0202021].

[27] M. Cvetič, H. Lü and C. N. Pope, M-theory, PP-Waves, Penrose Limits and Supernumerary Supersymmetries, Nucl. Phys. B644 (2002) 65-84; [hep-th/0203229].

[28] N. Iizuka, Supergravity, Supermembrane and M(atrix) model on PP-Waves, Phys. Rev. D 68 (2003) 126002; [hep-th/0211138].

[29] K. Dasgupta, M. M. Sheikh-Jabbari and M. Van Raamsdonk, Matrix perturbation theory for M-theory on a PP-wave, J. High Energy Phys. 05 (2002) 056; [hep-th/0205185].

[30] K. Dasgupta, M. M. Sheikh-Jabbari and M. Van Raamsdonk, Protected multiplets of M-theory on a plane wave, J. High Energy Phys. 09 (2002) 021; [hep-th/0207050].

[31] N. W. Kim and J. Plefka, On the spectrum of pp-wave matrix theory, Nucl. Phys. B643 (2002) 31; [hep-th/0207034].

[32] N. Kim and J. H. Park, Superalgebra for M-theory on a pp-wave, Phys. Rev. D 66 (2002) 106007; [hep-th/0207061].

[33] J. Michelson, Matrix theory of pp waves, in Quantum Theory and Symmetries: Proceedings of the 3rd International Symposium, (P. Argyres et. al. ed.) (World Scientific: New Jersey, 2004); [hep-th/0401050].

[34] J. H. Park, Supersymmetric objects in the M-theory on a pp-wave, J. High Energy Phys. 10 (2002) 032; [hep-th/0208161].

[35] J. Michelson, (Twisted) toroidal compactification of pp-waves, Phys. Rev. D 66 (2002) 066002; [hep-th/0203140].

[36] K. Sugiyama and K. Yoshida, Type IIA string and matrix string on pp-wave, Nucl. Phys. B644 (2002) 128; [hep-th/0208029].

[37] S. J. Hyun and H. J. Shin, $N=(4,4)$ type IIA string theory on pp-wave background, J. High Energy Phys. 10 (2002) 070; [hep-th/0208074].

[38] S. J. Hyun and H. J. Shin, Solvable $N=(4,4)$ type IIa string theory in plane-wave background and D-branes, Nucl. Phys. B654 (2003) 114; [hep-th/0210158]. 
[39] R. Gopakumar, String interactions in PP-waves, Phys. Rev. Lett. 89 (2002) 171601; [hep-th/0205174].

[40] S. R. Das, J. Michelson and A. D. Shapere, Fuzzy spheres in pp-wave matrix string theory, Phys. Rev. D 70 (2004) 026004; [hep-th/0306270].

[41] G. Bonelli, Matrix Strings in pp-wave backgrounds from deformed Super Yang-Mills Theory, J. High Energy Phys. 08 (2002) 022; [hep-th/0205213].

[42] H. Shin and K. Yoshida, One-loop flatness of membrane fuzzy sphere interaction in plane-wave matrix model, Nucl. Phys. B679 (2004) 99; [hep-th/0309258].

[43] Y. X. Chen and J. Shao, Giant graviton in type IIa pp-wave background, Phys. Rev. D 69 (2004) 106010; [hep-th/0310062].

[44] K. Furuuchi, E. Schreiber and G. W. Semenoff, Five-brane thermodynamics from the matrix model, hep-th/0310286.

[45] B. Janssen, Y. Lozano and D. Rodriguez-Gomez, Giant gravitons in $A d S_{3} \times S^{3} \times T^{4}$ as fuzzy cylinders, Nucl. Phys. B711 (2005) 392; [hep-th/0406148].

[46] H. K. Lee, T. McLoughlin and X. Wu, Gauge / gravity duality for interactions of spherical membranes in 11-dimensional pp-wave, CALT-68-2523, UK-04-21, hep-th/0409264.

[47] Y. Lozano and D. Rodriguez-Gomez, Fuzzy 5-spheres and pp-wave Matrix actions, FFUOV05/02, hep-th/0505073.

[48] E. Alvarez and P. Meessen, Newtonian M(atrix) cosmology, Phys. Lett. B 426 (1998) 282; [hep-th/9712136].

[49] D. Z. Freedman, G. W. Gibbons and M. Schnabl, Matrix cosmology, AIP Conf. Proc. 743 (2005) 286; [hep-th/0411119].

[50] M. Li, A class of cosmological matrix models, hep-th/0506260.

[51] M. Li and W. Song, Shock waves and cosmological matrix models, hep-th/0507185.

[52] G. Papadopoulos, J. G. Russo and A. A. Tseytlin, Solvable model of strings in a time-dependent plane-wave background, Class. Quant. Grav. 20 (2003) 969-1016; [hep-th/0211289].

[53] M. Blau, M O'Loughlin, G. Papadopoulos and A. A. Tseytlin, Solvable models of strings in homogeneous plane wave backgrounds, Nucl. Phys. B673 (2003) 57-97; [hep-th/0304198].

[54] M. Cvetič, H. Lü and C. N. Pope, Penrose Limits, PP-Waves and Deformed M2-branes, Phys. Rev. D 69 (2004) 046003; [hep-th/0203082].

[55] J. Figueroa-O'Farrill and G. Papadopoulos, Homogeneous Fluxes, Branes and Maximal Supersymmetry, J. High Energy Phys. 08 (2001) 036; [hep-th/0105308].

[56] J. P. Gauntlett and C. M. Hull, pp-waves in 11-dimensions with extra supersymmetry, J. High Energy Phys. 06 (2002) 013; [hep-th/0203255].

[57] J. G. Russo and A. A. Tseytlin, On solvable models of type IIB superstring in NS-NS and R-R plane wave backgrounds, J. High Energy Phys. 04 (2002) 021; [hep-th/0202179].

[58] J. Gomis, L. Motl and Andrew Strominger, PP-Wave / CFT $T_{2}$ Duality, J. High Energy Phys. 11 (2002) 016; [hep-th/0206166].

[59] J. Michelson, A pp-wave with 26 Supercharges, Class. Quant. Grav. 19 (2002) 5935-5949; [hep-th/0206204].

[60] N. Seiberg, Why is the Matrix Model Correct?, Phys. Rev. Lett. 79 (1997) 3577-3580; [hep-th/9710009].

[61] A. Sen, Do Branes on $T^{n}$ and Matrix Theory, Adv. Theor. Math. Phys. 2 (1998) 51-59; [hep-th/9709220].

[62] W. I. Taylor and M. Van Raamsdonk, Multiple D0-branes in weakly curved backgrounds, Nucl. Phys. B558 (1999) 63; [hep-th/9904095]. 
[63] J. McGreevy, L. Susskind and N. Toumbas, Invasion of the giant gravitons from anti-de Sitter space, J. High Energy Phys. 06 (2000) 008; [hep-th/0003075].

[64] M. T. Grisaru, R. C. Myers and Ø. Tafjord, SUSY and Goliath, J. High Energy Phys. 08 (2000) 040; [hep-th/0008015].

[65] A. Hashimoto, S. Hirano and N. Itzhaki, Large branes in AdS and their field theory dual, J. High Energy Phys. 08 (2000) 051; [hep-th/0008016].

[66] S. R. Das, A. Jevicki and S. D. Mathur, Giant gravitons, BPS bounds and noncommutativity, Phys. Rev. D 63 (2001) 044001; [hep-th/0008088].

[67] S. R. Das, S. P. Trivedi and S. Vaidya, Magnetic moments of branes and giant gravitons, J. High Energy Phys. 10 (2000) 037; [hep-th/0008203].

[68] S. R. Das, A. Jevicki and S. D. Mathur, Vibration modes of giant gravitons, Phys. Rev. D 63 (2001) 024013; [hep-th/0009019].

[69] N. D. Birrell and P. C. W. Davies, Quantum Fields in Curved Space, (Cambridge University Press: Cambridge, 1984).

[70] A. Strominger, Open string creation by S-branes, hep-th/0209090. 